\title{
Dinâmica da concentração de mercado na indústria brasileira, $1996-2003^{1}$ \\ Frederico Rocha ${ }^{2}$
}

\begin{abstract}
Resumo
O objetivo desse trabalho é estudar a mudança na concentração dos mercados da indústria brasileira de mineração e transformação entre 1996 e 2003, realizando uma decomposição do índice de Herfindahl-Hirschman em variação da distribuição do tamanho e do número de empresas. Os resultados obtidos sugerem que, na média, a indústria sofreu reduzida mudança no período; contudo, apontam que, em um grupo de indústrias, as mudanças no nível de concentração foram substanciais. Indicam também que a maior responsabilidade por essas mudanças está associada a alterações na desigualdade do tamanho, apesar de o período ter presenciado um forte movimento de entrada de novas empresas em diversos segmentos industriais.
\end{abstract}

Palavras-chave: Concentração de mercado; Indústria brasileira; Índice de Herfindahl-Hirschman; Brasil; Decomposição.

\section{Abstract \\ Dynamics of market concentration in the Brazilian industry, 1996-2003}

The aim of this paper is to study the changes in market concentration in the Brazilian mining and manufacturing industries that took place from 1996 to 2003. The paper decomposes the HerfindahlHirschman index into variations in the size distribution and the number of firms. The results show that the average concentration did not change greatly in the period analyzed; however, a small group of industries showed significant differences in concentration. In these cases, changes in size distribution appear to have more importance than changes in the number of firms.

Key words: Market concentration; Brazilian industry; Herfindahl-Hirschman index; Brazil; Decomposition.

JEL L11.

\section{Introdução}

Este artigo visa ao estudo da mudança na concentração dos mercados na indústria brasileira de mineração e transformação entre 1996 e 2003. Adotando o índice de Herfindahl-Hirschman como medida de concentração, o trabalho procura focar a atenção em dois temas:

(i) a variação do nível de concentração; e

(ii) a composição da variação entre distribuição do tamanho e número de empresas.

(1) Trabalho recebido em março de 2008 e aprovado em maio de 2009.

(2) Professor do Instituto de Economia da Universidade Federal Rural do Rio de Janeiro (UFRJ). Bolsista de Produtividade do CNPq (Conselho Nacional de Desenvolvimento Científico e Tecnológico), Rio de Janeiro, RJ, Brasil. E-mail: fred.rocha@ufrj.br. 
Frederico Rocha

O paradigma estrutura-conduta-desempenho (ECD) considera a concentração como aspecto central na definição das estruturas de mercado, representando um papel importante na definição do desempenho alocativo dos mercados. Os fatores estruturais são aqueles associados aos aspectos menos mutantes nas indústrias: concentração, barreiras à entrada, diversificação e verticalização das empresas, diferenciação de produto, concorrência internacional. A maior parte desses elementos apresenta interdependência e, de fato, a concentração parece estar relacionada com a maior parte dos fatores. Por exemplo, economias de escala devem definir concentração e barreiras à entrada em simultâneo; barreiras à entrada controlam o número de empresas afetando a concentração; verticalização pode servir como forma de controle de parcelas de mercado, entre outros. Assim, concentração passou, muitas vezes, a ser usada como uma medida objetiva que resume as características estruturais da indústria. Contudo, concentração é apenas uma entre inúmeras características que representam a estrutura de um determinado mercado (Scherer; Ross, 1990; Curry; George, 1983).

A evolução do paradigma ECD conduziu, no entanto, à constatação da endogeneidade de parte das variáveis de estrutura (Donsimoni et al., 1984). Esse debate se deu em duas frentes. No lado empírico, há dois tipos de evidência que questionam a linearidade do argumento do ECD. O primeiro trata da incapacidade das condições básicas de explicarem a concentração de maneira unidirecional, ainda que estejam correlacionadas. Isso advém de dois fatos estilizados: o nível de concentração de mercado é correlacionado com o nível de economias de escala no nível da unidade fabril e da firma; contudo, o nível de escala mínima está aquém do nível de concentração alcançado na maior parte dos mercados. O segundo argumento empírico é o fraco desempenho da concentração como elemento explicativo de variáveis de desempenho (Schmalensee, 1989).

Do ponto de vista teórico, a contestação parte de modelos de teoria dos jogos que mostram a prevenção à entrada e a proteção a parcelas de mercado das empresas estabelecidas como metas estratégicas da firma, definindo a endogeneidade da taxa de entrada e das parcelas de mercado das empresas (Tirole, $1988^{3}$; Dixit, 1982).

Assim, a concentração é não só uma variável que resume as características estruturais de um mercado, mas também uma variável que consolida informações sobre forças em operação em determinado mercado, incluindo variáveis estratégicas (no caso da ECD) e estocásticas (no caso da lei dos efeitos proporcionais e seus desdobramentos). Mais especificamente, sua evolução expressa as características da entrada líquida de empresas e a capacidade de firmas líderes protegerem e ampliarem suas parcelas de mercado. Nesse caso, conforme

(3) Ver capítulo 8. 
Donsimoni et al. (1984), índices de concentração podem ter uma função importante, principalmente se medidas de desempenho não podem ser adequadamente observadas.

O exame das mudanças na concentração e das forças que contribuíram para sua ocorrência parece ser relevante na evolução recente da indústria brasileira. Entre 1996 e 2003, a indústria de mineração e transformação apresentou elevadas taxas de entrada de empresas, tendo o número de empresas se elevado em pouco menos de 30\%. Em simultâneo, teve lugar uma forte atividade de fusões e aquisições, disseminada em grande parte dos setores da indústria. Além disso, as grandes empresas promoveram renovação em seu maquinário, em decorrência da abertura comercial. Nesse caso, ao adotar métodos mais eficientes, tais empresas ganharam participação em seus mercados de atuação. Portanto, é de se esperar mudanças na concentração dos setores, de acordo com a combinação entre as forças de desconcentração provenientes da entrada líquida de empresas, e aquelas concentradoras, associadas a mudanças internas nas parcelas de mercado das empresas (Rocha, 2004; Ferreira; Rossi, 2003; Schor, 2003).

O restante do trabalho está dividido em três seções. Na seção 1, faz-se uma avaliação do índice de Herfindahl-Hirschman como medida do nível de concentração de mercados e procura-se explorar suas principais potencialidades e limitações. A seção 2 é voltada para a exposição da base de dados, enquanto a seção 3 apresenta uma análise dos resultados. Por fim são exploradas as principais conclusões do artigo.

\section{Contexto analítico}

\section{1 Índices de concentração e o índice de Herfindahl-Hirschman}

Hall e Tideman (1967) e Hannah e Kay (1977) listam uma série de características que devem ser atendidas por uma medida de concentração. Ambos os trabalhos partem do princípio de que as medidas de concentração devem tomar como base as parcelas de mercado de cada firma, além de considerar concentração uma função da desigualdade das parcelas de mercado e do número de firmas. ${ }^{4}$

(4) Podem, portanto, ser listadas as seguintes características desejáveis em índices de concentração: (i) ser unidimensional, implicando não produzir ambiguidades na comparação entre dois mercados distintos; (ii) independência da dimensão do mercado; (iii) transferências de parcelas de mercado de uma firma de menor parcela para uma firma de maior parcela devem afetar positivamente o índice de concentração; (iv) um incremento na concentração cumulativa da firma $i$, sendo as firmas listadas em ordem decrescente de tamanho de $1,2, \ldots, i, \ldots n$ deverá aumentar a concentração do mercado; (v) fusões entre duas firmas devem aumentar o nível de concentração do mercado; (vi) a entrada de novas firmas abaixo de um tamanho arbitrário deve reduzir a concentração do mercado; (vii) se as firmas são divididas em $K$ partes, então o índice deve indicar uma concentração $1 / K$; (viii) quando a indústria é dividida em $n$ firmas de igual tamanho, então o nível de concentração deve ser uma função decrescente de $n$; e (ix) se $s_{i}$ é a parcela de mercado da firma entrante e $s_{i}$ é decrescente, então a variação do índice de concentração também deve ser decrescente.

Economia e Sociedade, Campinas, v. 19, n. 3 (40), p. 477-498, dez. 2010. 
As razões de concentração são os índices de mais antiga utilização. Ele é definido como $C R_{k}=\sum_{i=1}^{k} s_{i}$, em que $s_{i}$ é a parcela de mercado da $i$-ésima empresa e $i=(1, . ., k, . . n)$ ordenado de maneira decrescente. O problema do índice é que trata apenas de um ponto na curva de concentração cumulativa. Assim, existe perda de informação na avaliação da concentração por intermédio desse procedimento (Curry; George, 1983, p. 207).

Grande parte dos índices de concentração utilizados na literatura atende às propriedades listadas acima. Hannah e Kay (1977) designam uma família de índices que mantêm essas características, designada por: ${ }^{5}$

$$
H K(\alpha)=\left\{\begin{array}{l}
\left(\sum_{i}^{n} s_{i}^{\alpha}\right)^{\left(\frac{1}{\alpha-1}\right)} \text { para } \alpha \neq 1 \\
\exp \left[\sum_{i}^{n} s_{i} \ln s_{i}\right] \text { para } \alpha=1
\end{array}\right.
$$

em que $s_{i}$ é a parcela de mercado da firma $i$. Adotando-se $\alpha=2$, obtém-se o índice de Herfindahl-Hirschman $(H)$ :

$$
H=\sum_{i}^{n} s_{i}^{2}
$$

A escolha do valor de $\alpha$ é arbitrária. Quanto maior seu valor, maior o peso concedido às empresas de maior porte. Portanto, em $H$, ao elevar ao quadrado a parcela de mercado de cada empresa, o índice concede maior peso às firmas maiores.

A principal justificativa para a adoção do índice $H$ é a possibilidade de estabelecimento de relação direta entre o seu valor e o índice de Lerner, desde que adotadas as hipóteses comportamentais Nash-Cournot (Hay; Morris, 1989, p. 211), isto é, $\frac{H}{|\varepsilon|}=\frac{p-\overline{C M g}}{p}$, em que $|\varepsilon|$ é a elasticidade da demanda, $p$ o preço, e $\overline{C M g}$ o custo marginal médio do mercado, sendo que o lado direito da equação representa o índice de Lerner.

$\mathrm{O}$ índice $H$ pode ser decomposto em duas parcelas para analisar concentração, ou seja, o grau de desigualdade das parcelas de mercado e o número de firmas no mercado. Denotando-se $\sigma^{2}$ por variância, tem-se $\sigma^{2}=\frac{1}{n} \sum_{i}\left(\frac{1}{n}-s_{i}\right)^{2}$,

(5) Na verdade, Hannah e Kay (1977) exploram o número equivalente $\frac{1}{H K}(\alpha)=\left(\sum_{i}^{n} s_{i}^{\theta}\right)^{\left(\frac{1}{1-\alpha}\right)}$. 
em que $n$ é o número de firmas e $1 / n$ é a média das parcelas de mercado. Como consequência:

$$
H=n \cdot \sigma^{2}+\frac{1}{n}
$$

$\mathrm{e}$

$$
H=\frac{1+(C V)^{2}}{n}
$$

em que $\frac{1}{n}$ é a média e $C V=n \sigma$ o coeficiente de variação e, portanto, um indicador da distribuição de tamanho das firmas. ${ }^{6}$ Percebe-se, ainda, a partir de (4) que variações no número de empresas influenciam a dispersão do tamanho, na medida em que alteram a média. Portanto, um aumento de $n$, mantida constante a variância, pode resultar em incremento da desigualdade. O grande problema é conhecer o tamanho relativo da entrada.

Denotando-se o numerador da equação (4) por $\varphi\left(C V^{2}\right)$, pode-se obter $\frac{H_{1}}{H_{0}}=\left(1+g_{H}\right)=\frac{\left(1+g_{\varphi}\right)\left(\varphi\left(C V^{2}\right)\right)}{\left(1+g_{n}\right) n} \mathrm{e}$, com um pouco de álgebra, chega-se à equação dinâmica do índice $H$ :

$$
\underbrace{g_{H}}_{\begin{array}{c}
\text { Taxa de Crescimento } \\
\text { do Herfindahl }
\end{array}}=\underbrace{g_{\varphi}}_{\begin{array}{c}
\text { Efeito } \\
\text { Desigualdade }
\end{array}}-\underbrace{g_{n}}_{\begin{array}{c}
\text { Efeito } \\
\text { Entrada Líquida }
\end{array}}-\underbrace{\frac{g_{n}\left(g_{\varphi}-g_{n}\right)}{1+g_{n}}}_{\text {Efeito Interação }}
$$

A equação (5) decompõe a variação do índice de Herfindahl-Hirschman em três parcelas. ${ }^{7}$ A primeira é associada à variação da dispersão dos tamanhos

(6) Resultado semelhante é obtido por Bajo e Salas (2002), a partir de um índice geral de desigualdade

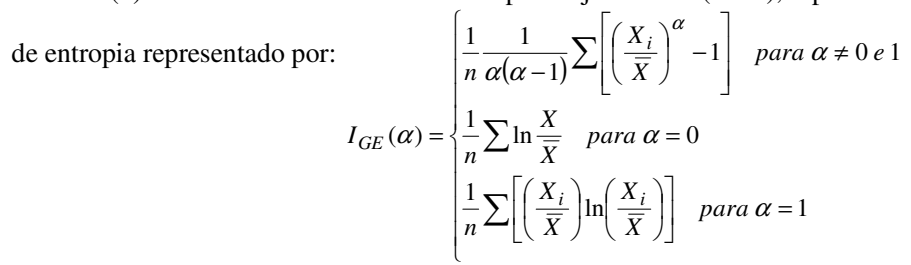

em que $X_{i}$ é a renda da firma $i$ e $\bar{X}$ é a renda média das firmas da indústria. A partir de $\alpha=2$ e substituindo em (2), eles obtêm $H=\frac{1+2 I_{G E}(2)}{n}$.

(7) Essa equação deve ser comparada com $g_{H}=g_{\varphi}-g_{n}$ de Bajo e Salas (2002), obtida a partir da equação (3), passada para base logarítmica e derivada em relação ao tempo. O termo adicional presente em (4) é o efeito interação que advém do fato de se estar trabalhando com tempo discreto, ao invés de contínuo. De fato, Bajo e Salas (2004) testam a equação para a economia espanhola, por meio de variação contínua do tempo. O resultado apresenta um valor residual, equivalente ao terceiro termo da equação (4). 
das empresas da indústria, a segunda reflete a variação do número de empresas e a terceira é um termo de interação, que assume valor negativo sempre que $g_{\varphi}>g_{n}$, e positivo se o inverso for verdadeiro.

\subsection{Dinâmica dos Índices de Concentração}

A equação (5) procura dar conta de uma crítica frequentemente levantada quanto ao funcionamento dos índices de concentração: seu caráter eminentemente estático. Isso se deveria à falha em captar adequadamente a habilidade das firmas líderes deterem a entrada ou impedirem a deterioração de suas fatias de mercado. Contudo, grande parte dos trabalhos avaliados demonstra elevada correlação entre variação de medidas de concentração e variáveis representativas de poder de mercado. Grossack (1965) e Gort (1963) procuram medir poder de mercado pela capacidade de uma empresa manter ou ampliar sua parcela de mercado. A medida alternativa escolhida para avaliação é o coeficiente da regressão das parcelas de mercado do período 0 sobre as parcelas de mercados no período 1 , representada por $b=\frac{\sum_{i=1}^{n} x_{i, 0} x_{i, 1}}{\sum_{i=1}^{n} x_{i, 0}^{2}}$, sendo $x_{i, t}=\left(s_{i, t}-\bar{s}_{i, t}\right)$, em que $s_{i, t}$ é a parcela de mercado da firma $i$, no período $t$, e $\bar{s}_{i t}$ a média das parcelas de mercado. Se $b$ é maior do que 1 , as grandes empresas do período 0 terão ampliado suas parcelas de mercado. Se $b$ é menor do que 1 , terão diminuído. De maneira equivalente, por produto dos momentos,

$$
b=r \frac{\sigma_{1}}{\sigma_{0}}
$$

em que $r$ é o coeficiente de correlação entre as parcelas de mercado dos dois períodos e $\sigma_{t}$ desvio padrão no período $t$. A partir da equação (3), tem-se que $\sigma=\sqrt{\frac{H}{n}-\frac{1}{n^{2}}}$. De maneira que:

$$
b=r \frac{\sqrt{\frac{H_{1}}{n_{1}}-\frac{1}{n_{1}^{2}}}}{\sqrt{\frac{H_{0}}{n_{0}}-\frac{1}{n_{0}^{2}}}}
$$

A partir da equação (7) entende-se que a medida utilizada por Grossack (1965) para representar utilização do poder de mercado é uma função do índice de Herfindahl-Hirschman. Deve-se acrescentar que, na aplicação empírica em mais de 200 setores da indústria de transformação dos EUA, Grossack (1965) encontra 
apenas dois setores com $r<0,8$, o que torna grandes variações do desvio padrão suficiente para obter valores de $b$ maiores do que 1 .

\section{Base de dados}

Esse trabalho utiliza a receita líquida de vendas por empresa da Pesquisa Industrial Anual do IBGE (PIA), no período de 1996 a 2003, como variável para mensuração das parcelas de mercado das empresas. Arbitrou-se por restringir a análise ao nível de três dígitos da Classificação Nacional de Atividades Econômicas.

A classificação de três dígitos da CNAE inclui 106 setores industriais. Contudo, para três setores não há disponibilidade de dados na PIA (setores com menos de três empresas). Logo, o trabalho aqui realizado avaliou 103 setores. A utilização de fontes estatísticas oficiais estabelece limitações quanto à delimitação do mercado. O recorte do mercado relevante é definido pelo tipo de classificação setorial utilizada. Como enfatizado por Hay e Morris (1991, p. 207), as classificações de organismos estatísticos oficiais estão normalmente formuladas pelo critério de similaridade do lado da produção, e não por critérios de substituição da demanda. A Classificação Nacional de Atividades Econômicas (CNAE), utilizada pelo IBGE, não é exceção.

Opções por níveis de agregação mais elevados estão sujeitas ao erro de se definir o mercado relevante de maneira ampla demais, subestimando a concentração. Ademais, principalmente no caso brasileiro - em que o grau de diversificação das firmas é reduzido - o locus da concorrência deverá ser determinado em níveis de agregação menos elevados. O Censo Cadastro de 1994 apresenta dados sobre a diversificação das atividades empresariais na indústria de transformação. Cerca de $2 \%$ das empresas atuam em mais de um grupo, sendo responsáveis por cerca de $18 \%$ da receita total da indústria de transformação. Assim, a utilização do grupo como unidade de análise indica um risco inferior a $18 \%$ de se estar somando uma receita ao setor errado. A utilização de classe (quatro dígitos) conduz a possíveis erros na alocação da receita. O critério de definição da receita em determinado setor está associado à declaração da principal atividade da unidade investigada pelo IBGE. Assim, a escolha da classe poderá incluir o erro de definir uma receita muito ampla para um determinado setor e muito estreita para outro.

A Pesquisa Industrial Anual é censitária para empresas com trinta ou mais empregados e uma base amostral para empresas de cinco a vinte e nove

empregados, não cobrindo empresas com menos de cinco empregados. Nesse caso, é importante ressaltar que a composição do estrato amostral está baseada no Cadastro Central de empresas do IBGE, atualizado anualmente, tendo por base as 
diversas pesquisas realizadas pelo IBGE e as informações do Registro Anual de Informações Sociais (RAIS) e do Cadastro Geral de Empregados e Desempregados (CAGED). Essa característica é particularmente importante quando se está lidando com entrada e saída de empresas nessas faixas de tamanho, visto que o número de empresas de cada setor, em suas regiões amostrais, será dependente da qualidade dos registros administrativos.

A partir do índice $H$ e do número de empresas para os setores a três dígitos da PIA, este trabalho realizará a decomposição proposta na equação (5), analisando as mudanças no índice $H$ e separando o papel representado pelas mudanças na desigualdade do tamanho das empresas e pelo papel representado pela mudança no número de empresas.

\section{Resultados}

A primeira pergunta a ser respondida neste trabalho é se o período em questão registrou incremento de concentração na indústria de mineração e transformação no Brasil. Deve-se notar que a concentração média, quando medida a três dígitos, não é muito elevada. A Tabela 1 apresenta a evolução da concentração média da indústria para os anos de 1996, 2000 e 2003. O número equivalente de empresas, representado por $\frac{1}{H}$, é próximo a dez, sugerindo que o nível de concentração médio dos mercados industriais é equivalente ao de dez empresas de igual porte atuando no mercado. A razão de concentração sugere um valor compatível ao obtido pelo índice $H$, na medida em que as quatro maiores empresas do mercado, em média, alcançam pouco mais de $40 \%$ do mercado. Em média, portanto, não parece ter ocorrido elevada concentração no país.

Quando se avaliam os setores em diferentes faixas de concentração, o quadro parece confirmar que poucos setores registraram grandes saltos de concentração. A Tabela 2 mostra a distribuição das indústrias por faixas de concentração, de acordo com o índice $H$. As três primeiras faixas de concentração foram elaboradas a partir dos critérios adotados pelo Mergers Guidelines da Federal Trade Comissions dos EUA. ${ }^{8}$ A última faixa de concentração se refere a estruturas de mercado com número equivalente de empresas inferior a 2. Fica claro que a grande maioria das indústrias apresenta baixo nível de concentração,

(8) Na faixa de $\mathrm{H}<0,1$, os atos de concentração não são submetidos à averiguação da FTC; em faixas de concentração de 0,1 a 0,18 , os atos de concentração que impliquem aumento do índice maior ou igual a 0,01 são objetos de averiguação; a partir de 0,18 , atos de concentração que impliquem variações de $H$ iguais ou superiores a 0,05 são averiguados. 
não fazendo parte do que seria a faixa regulável para fusões de acordo com as autoridades de defesa da concorrência. ${ }^{9}$

Tabela 1

Evolução da concentração média na indústria de mineração e transformação, Brasil, 1966, 2000, 2004*

\begin{tabular}{l|c|c|c}
\hline & 1996 & 2000 & 2003 \\
\hline H médio & 0,0934 & 0,1057 & 0,0974 \\
\hline$\varphi$ médio & 26,3729 & 34,9284 & 38,2085 \\
\hline n médio & 108156 & 124778 & 137547 \\
\hline CR4 médio & 0,4118 & 0,4296 & 0,4202 \\
\hline
\end{tabular}

* n é a taxa de crescimento do número de empresas, $\mathrm{H}$ é o índice de Herfindahl-Hirschman, $\varphi$ é o indicador de variância do tamanho e CR4, a razão de concentração das quatro maiores empresas.

Fonte: Elaboração própria a partir de IBGE - Pesquisa Industrial Anual.

Percebe-se também estabilidade no nível de concentração médio ao longo do tempo. Das 103 indústrias analisadas, 77 permaneciam, em 2003, na mesma faixa de concentração em que se encontravam em 1996. De fato, o teste de diferença de médias dos índices de concentração Hirschman-Herfindahl em 1996 e 2003 obtém uma estatística $\mathrm{z}=0,06$, mantendo a hipótese de médias iguais. Ademais, a correlação entre o índice de Herfindahl-Hirschman de 1996 e o de 2003 é de 0,795 , e significativa a $1 \%$.

Tabela 2

Distribuição do número de indústrias por faixas de concentração, Brasil, 1996 e 2003

\begin{tabular}{|c|c|c|c|c|c|}
\hline \multirow[b]{2}{*}{ Faixa de Concentração 1996} & \multicolumn{4}{|c|}{ Faixa de Concentração 2003} & \multirow[b]{2}{*}{$\begin{array}{l}\text { Total } \\
\text { geral }\end{array}$} \\
\hline & $\begin{array}{c}\text { H menor } \\
\text { que } 0,1\end{array}$ & $0,1<\mathrm{H}<0,18$ & $0,18<\mathrm{H}<0,5$ & $\begin{array}{c}\text { Maior que } \\
0,5\end{array}$ & \\
\hline $\mathrm{H}$ menor que 0,1 & 66 & 10 & & & 76 \\
\hline $0,1 \leq \mathrm{H}<0,18$ & 1 & 5 & 7 & & 13 \\
\hline $0,18 \leq \mathrm{H}<0,5$ & 1 & 5 & 5 & 1 & 12 \\
\hline 5000 ou mais & & 1 & & 1 & 2 \\
\hline Total geral & 68 & 21 & 12 & 2 & 103 \\
\hline
\end{tabular}

Fonte: Elaboração própria a partir de IBGE - Pesquisa Industrial Anual.

Contudo, não é desprezível o número de indústrias que saltaram de faixa de concentração. Oito indústrias passaram para faixas de concentração inferiores e 18 foram para superiores. Portanto, a estabilidade média encontrada na Tabela 1

(9) A Tabela 20 usa o critério de defesa da concorrência. É importante refletir, contudo, que esse critério deve ser aplicado com a adequada definição de mercado relevante, trabalho não realizado aqui. Note-se que o nível de concentração médio encontrado a três dígitos (mais ou menos 100 setores) é semelhante ao índice de concentração encontrado nos EUA para setores desagregados a quatro dígitos (mais de 200 setores industriais), de acordo com Pryor (2002) a partir de estatísticas do Censo norte-americano. 
parece ser consequência de compensações de tendências diferenciadas entre os setores. O Gráfico 1 0mostra que, em 54 setores, houve aumento da concentração, enquanto em 49 deles houve diminuição da concentração, medida pelo índice $H$. Em 44 setores, as variações, para mais ou para menos, são desprezíveis - ou seja, menos de 0,01 ponto do índice $H$. Em 28 setores a concentração variou mais 0,05 ponto do índice $H$ e, em 10 deles, mais de 0,1 ponto. Nesses setores, as variações na concentração estão longe de poderem ser consideradas desprezíveis.

O quadro parece um pouco mais dinâmico quando se procura verificar os diferentes componentes da concentração, isto é, quando se analisam em separado o número de empresas e a distribuição de tamanho de cada indústria. Houve um aumento de $27 \%$ no número de empresas ${ }^{10}$ e um forte incremento da estatística $\varphi$, quer dizer, aumento na dispersão de tamanho das empresas da indústria (ver Tabela 10).

Setenta e cinco dos 103 setores avaliados apresentam sinal positivo para a variação da componente de desigualdade - $\varphi$ (ver Tabela 30). Ao mesmo tempo, a grande maioria dos setores apresenta entrada líquida de empresas. Em apenas 19 dos 103 setores não há entrada líquida de empresas. Já o termo interação apresenta sinal negativo, ou seja, a taxa de crescimento da desigualdade é superior à taxa de crescimento do número de empresas, em 55 casos, e sinal positivo, ou seja, a taxa de crescimento do número de empresas supera a taxa de crescimento da desigualdade em 48 setores.

Gráfico 1

Distribuição dos setores a três dígitos da CNAE de acordo com a dimensão da variação da concentração segundo o Índice de Herfindahl-Hirschman

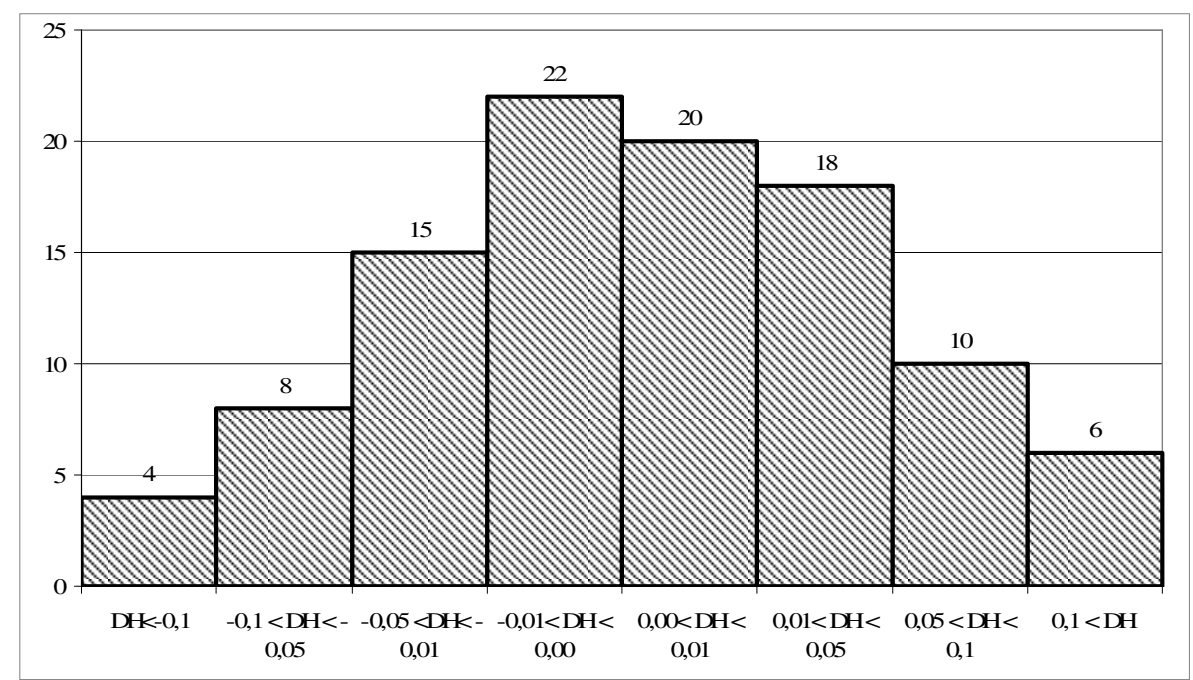

Fonte: Elaboração própria a partir de IBGE - Pesquisa Industrial Anual.

(10) A taxa de crescimento do número de empresas foi superior a $20 \%$ em 61 setores. 
Em 74 setores os sinais do efeito desigualdade e do efeito entrada líquida são invertidos; desses, sessenta e cinco apresentam crescimento da desigualdade e do número de empresas, enquanto em oito há decréscimo da desigualdade e do número de empresas. $O$ índice de correlação de Pearson entre o efeito desigualdade e o efeito entrada líquida é de -0,234 e significativo a 5\%; ou seja, na maior parte dos casos, quando há uma forte entrada de empresas, ocorre um aumento da desigualdade, compensando o efeito global da entrada de empresas sobre o índice de concentração. ${ }^{11}$ Uma das explicações para esse comportamento pode ser a entrada predominante de empresas de pequeno porte. Em 1996, as empresas com 100 ou mais empregados representavam 7,2\% do total de empresas da indústria de transformação e mineração. Essa participação caiu para 5,9\%, em 2003. Apenas $1,3 \%$ das entrantes tinham 100 ou mais empregados e $83 \%$ tinham menos de 30 empregados. A partir da equação (3), sabe-se que um aumento em $n$, mantendo-se o desvio padrão constante, só afetará positivamente o índice de concentração se o desvio padrão das parcelas de mercado for maior do que a média da parcela de mercado, o que requer que o coeficiente de variação equação (4) - seja menor do que 1 (o que é bastante improvável). ${ }^{12}$ De fato, isso não ocorre em nenhum dos 103 setores analisados, sendo uma consequência do efeito indireto que a entrada exerce sobre a desigualdade.

Tabela 3

Sinal dos termos da equação de variação do $H$ nos 103 setores da indústria de mineração e transformação

\begin{tabular}{|c|c|c|c|c|c|c|c|}
\hline \multirow{4}{*}{$\begin{array}{l}\text { Faixas de Variação } \\
\text { de } H\end{array}$} & \multicolumn{7}{|c|}{ Efeito Entrada Líquida } \\
\hline & \multicolumn{3}{|c|}{ Negativo } & \multicolumn{3}{|c|}{ Positivo } & \multirow{3}{*}{ Total } \\
\hline & \multicolumn{2}{|c|}{$\begin{array}{l}\text { Sinal do Efeito } \\
\text { Desigualdade }\end{array}$} & \multirow[t]{2}{*}{ Total } & \multicolumn{2}{|c|}{$\begin{array}{l}\text { Sinal do Efeito } \\
\text { Desigualdade }\end{array}$} & \multirow[t]{2}{*}{ Total } & \\
\hline & Negativo & Positivo & & Negativo & Positivo & & \\
\hline$\Delta \mathrm{H}<-0,1$ & 3 & 1 & 4 & & & & 4 \\
\hline$-0,1<\Delta \mathrm{H}<-0,05$ & 3 & 5 & 8 & & & & 8 \\
\hline$-0,05<\Delta \mathrm{H}<-0,01$ & 10 & 4 & 14 & 1 & & 1 & 15 \\
\hline$-0,01<\mathrm{DH}<0,00$ & 3 & 16 & 19 & 3 & & 3 & 22 \\
\hline Total Negativo & 19 & 26 & 45 & 4 & & 4 & 49 \\
\hline $0,00<\mathrm{DH}<0,01$ & & 16 & 16 & 2 & 2 & 4 & 20 \\
\hline $0,01<\mathrm{DH}<0,05$ & & 12 & 12 & & 6 & 6 & 18 \\
\hline $0,05<\mathrm{DH}<0,1$ & & 7 & 7 & & 3 & 3 & 10 \\
\hline $0,1<\mathrm{DH}$ & & 4 & 4 & & 2 & 2 & 6 \\
\hline Total Positivo & 0 & 39 & 39 & 5 & 10 & 15 & 54 \\
\hline Total Geral & 19 & 65 & 84 & 9 & 10 & 19 & 103 \\
\hline
\end{tabular}

Fonte: Elaboração própria a partir de IBGE - Pesquisa Industrial Anual.

(11) Deve-se destacar que a correlação entre o efeito desigualdade e a variação do índice $H$ é mais forte do que a correlação entre o efeito entrada líquida e a variação do índice $H$, respectivamente 0,78 e 0,285 .

(12) Derivando (3), com respeito a $n$, tem-se: $\frac{\partial H}{\partial n}=\sigma^{2}-\frac{1}{n^{2}}<0 \Rightarrow \sigma^{2}<\frac{1}{n^{2}}$. Argumento semelhante é desenvolvido por Laderman (1995) para o caso específico do sistema bancário urbano. 
O Gráfico 2 0apresenta os efeitos distribuição, entrada líquida e interação distribuídos por setor da indústria de mineração e transformação. ${ }^{13}$ Os setores estão ordenados pela taxa de crescimento do índice $H$, de modo que, à esquerda, têm-se os setores com maiores taxas de decréscimo da concentração e, à direita, os setores com maiores taxas de crescimento da concentração. A observação do Gráfico 2 0permite perceber que a relativa estabilidade dos índices de concentração, denotada pela observação do crescimento do índice $H$, parece ser resultado de um quadro bastante dinâmico sob o ponto de vista de entrada e saída de empresas e ganhos e perdas de parcelas de mercado. Na maioria dos casos, o efeito entrada líquida assume valor negativo, e o efeito desigualdade, positivo.

Entre os setores de maior crescimento da concentração, todos apresentam efeito desigualdade positivo, ou seja, em todos eles houve aumento da desigualdade e apenas quatro apresentam o efeito entrada líquida positivo. Tanto o aumento da desigualdade quanto a redução do número de empresas pode ser explicado por mudanças patrimoniais. A Tabela 4 deixa transparecer que entre os setores que mais se concentraram estão alguns dos que mais sofreram intervenções de fusões e aquisições. Isso ocorreu nos quatro setores de alimentos listados. Antártica e Brahma realizaram uma importante fusão no segmento de Bebidas, enquanto Cargill e Bunge compraram empresas de pequeno porte no segmento de Produção de óleos, gorduras vegetais. A Bunge também atuou no segmento de outros alimentos na compra de empresas associadas a panificação (setor 1581) e biscoitos (setor 1582). Em outros produtos alimentícios, algumas empresas multinacionais típicas de laticínios compraram empresas de biscoitos. ${ }^{14}$ Esse movimento fez parte de um processo de internacionalização da indústria de alimentos - não particular do Brasil, mas atuante sobre a América Latina como um todo - e já identificado em outros textos (ver Rocha; Kupfer, 2002; Mortimore; Peres, 2001). Contudo, esse fenômeno não esteve limitado a Alimentos. Em Máquinas e equipamentos agrícolas, algumas fusões internacionais auxiliam na explicação da concentração. Nos demais segmentos com grande elevação do nível de concentração outros eventos parecidos podem ser registrados.

A importância do processo de fusões e aquisições deve ser, no entanto, relativizada, em razão das diferentes formas que esse processo pode tomar: desmembramento, entrada em mercados, entre outros. Um exemplo desse argumento é a presença de Laticínios - indústria em que houve forte atividade de fusões e aquisições - entre os setores que mais se desconcentraram. ${ }^{15}$ No entanto,

(13) Os dados utilizados para a composição do gráfico estão no Anexo deste artigo.

(14) Deve-se ressaltar que a maior parte dessas informações foram obtidas da Thomson Financial Securities Data.

(15) Sugere-se a leitura de Liebeskind, Opler e Hatfield (1996) e Rocha (2004) para uma exposição desse ponto, com resultados empíricos para EUA e Brasil, respectivamente. 
a partir dessa descrição, evidencia-se a importância dos processos de reestruturação produtiva nos segmentos que mais se concentraram. Isso também é evidente em Máquinas para escritório. Esse setor teve uma das maiores taxas de crescimento da receita líquida de vendas no período. No entanto, o número de empresas decresceu. Assim, a parcela de mercado de empresas eliminadas foi ocupada, de maneira desigual, por empresas pertencentes ao setor.

Assim, mudanças na desigualdade parece ser o principal fator explicativo da variação na concentração. Vale acrescentar que em apenas dois entre os 54 setores que tiveram variações positivas do grau de concentração houve efeito desigualdade negativo, mesmo assim com taxas de variação muito pequenas (ver também o Anexo).

Tabela 4

Decomposição da taxa de crescimento do Índice $H$ dos dez setores que mais se desconcentraram e dos dez setores que mais se concentraram

\begin{tabular}{|c|c|c|c|}
\hline Setor & $\begin{array}{c}\text { Efeito } \\
\text { Desigualdade }\end{array}$ & $\begin{array}{c}\text { Efeito } \\
\text { Entrada } \\
\text { Líquida }\end{array}$ & $\begin{array}{c}\text { Efeito } \\
\text { Interação }\end{array}$ \\
\hline \multicolumn{4}{|c|}{ Setores que mais se Concentraram } \\
\hline Produção de óleos, gorduras vegetais & 1,2039 & $-0,0964$ & $-0,0974$ \\
\hline Fabricação de tratores e de máq. e equip. agrícolas & 1,6746 & $-0,2324$ & $-0,272$ \\
\hline Forjaria, estamparia, metalurgia do pó e serviços & 1,7652 & $-0,2644$ & $-0,3138$ \\
\hline Tecelagem - inclusive fiação e tecelagem & 1,0484 & 0,1145 & 0,1503 \\
\hline Fabricação e refino de açúcar & 1,2386 & 0,0719 & 0,1015 \\
\hline Fundição & 1,707 & $-0,1097$ & $-0,1579$ \\
\hline Extração de minerais metálicos não-ferrosos & 0,9584 & 0,3333 & 0,6459 \\
\hline Fabricação de bebidas & 3,2909 & $-0,2776$ & $-0,6548$ \\
\hline Fabricação de outros produtos alimentícios & 2,5094 & 0,0157 & 0,0402 \\
\hline Fabricação de máquinas para escritório & 1,1581 & 0,4138 & 1,1096 \\
\hline \multicolumn{4}{|c|}{ Setores que mais se Desconcentraram } \\
\hline Reciclagem de sucatas metálicas & 0,1807 & $-3,75$ & 2,8179 \\
\hline Construção e reparação de embarcações & $-0,6123$ & $-0,2977$ & 0,2088 \\
\hline Produção de ferro-gusa e de ferroligas & 0,2974 & $-3,2632$ & 2,2701 \\
\hline Reciclagem de sucatas não-metálicas & 0,9664 & $-5,1176$ & 3,4726 \\
\hline Laticínios & $-0,4934$ & $-0,4739$ & 0,311 \\
\hline Fabricação de fios, cabos e condutores elétricos & $-0,6018$ & $-0,1009$ & 0,0644 \\
\hline Fabricação de tanques, caldeiras e reservatórios & $-0,3765$ & $-0,2921$ & 0,1511 \\
\hline Fabricação de pilhas, baterias e acumuladores & $-0,3273$ & $-0,36$ & 0,1819 \\
\hline Recondicionamento ou recuperação de motores & $-0,599$ & 0,2088 & $-0,103$ \\
\hline Extração de outros minerais não-metálicos & $-0,317$ & $-0,3233$ & 0,1564 \\
\hline
\end{tabular}

Fonte: Elaboração própria a partir de IBGE - Pesquisa Industrial Anual. 
Frederico Rocha

Nos dez setores que mais se desconcentraram relativamente, o efeito desigualdade também aparenta ter representado um papel relevante (Tabela 40). Ele é negativo em sete setores. Desses, cinco são superiores em módulo ao efeito entrada líquida. Em apenas três setores ele apresenta sinal positivo. Dois desses três casos são segmentos ligados à reciclagem. São setores muito novos, com empresas de pequeno porte e cuja entrada de empresas pode estar ocorrendo sem a redução do porte médio da indústria. ${ }^{16}$

Um ponto interessante a ser explorado é o setor de Laticínios, talvez um dos recordistas em fusões e aquisições no período e, contudo, com forte decréscimo da concentração. A forte entrada de empresas acontece por um movimento de concentração da produção em cooperativas de leite. Grande parte da produção de leite era feita por microestabelecimentos (com menos de cinco empregados). A formação e a ampliação do tamanho de cooperativas produtoras de leite trouxeram como efeito uma entrada de empresas na amostra da PIA. Contudo, conforme ressaltado acima, a entrada de empresas não garante redução da concentração. Assim, a ampliação do tamanho das cooperativas também garantiu maior igualdade entre o tamanho das empresas.

Isso sugere que a entrada de empresas no período, apesar de forte, não é condição necessária para uma redução forte na taxa de concentração. $O$ efeito entrada líquida não parece, portanto, ser determinante da configuração das estruturas de mercado. Dos sete setores de maior taxa de variação do número de empresas, apenas quatro tiveram $\Delta H$ negativo, enquanto os outros três apresentaram aumento da concentração. Assim, mesmo quando a entrada é intensa, a compensação fornecida pelo aumento da desigualdade e pela interação entre os dois efeitos parece poder reverter a situação.

O efeito interação só parece ser relevante nos extremos e principalmente nos setores de maior taxa de desconcentração. Nos setores de baixa variação da concentração há reduzida relevância.

(16) O tamanho médio das empresas da indústria de transformação e mineração, medido pelo critério de receita líquida de vendas, é de $\mathrm{R} \$ 3,3$ milhões. O setor de reciclagem de sucatas não-metálicas tem em um tamanho médio de $\mathrm{R} \$ 400$ mil e o de reciclagem de sucatas metálicas de $\mathrm{R} \$ 2,2$ milhões, (dados da PIA 1996). 
Gráfico 2

Efeitos distribuição, entrada líquida e interação nos 103 setores ordenados por taxa de crescimento do Índice $H$

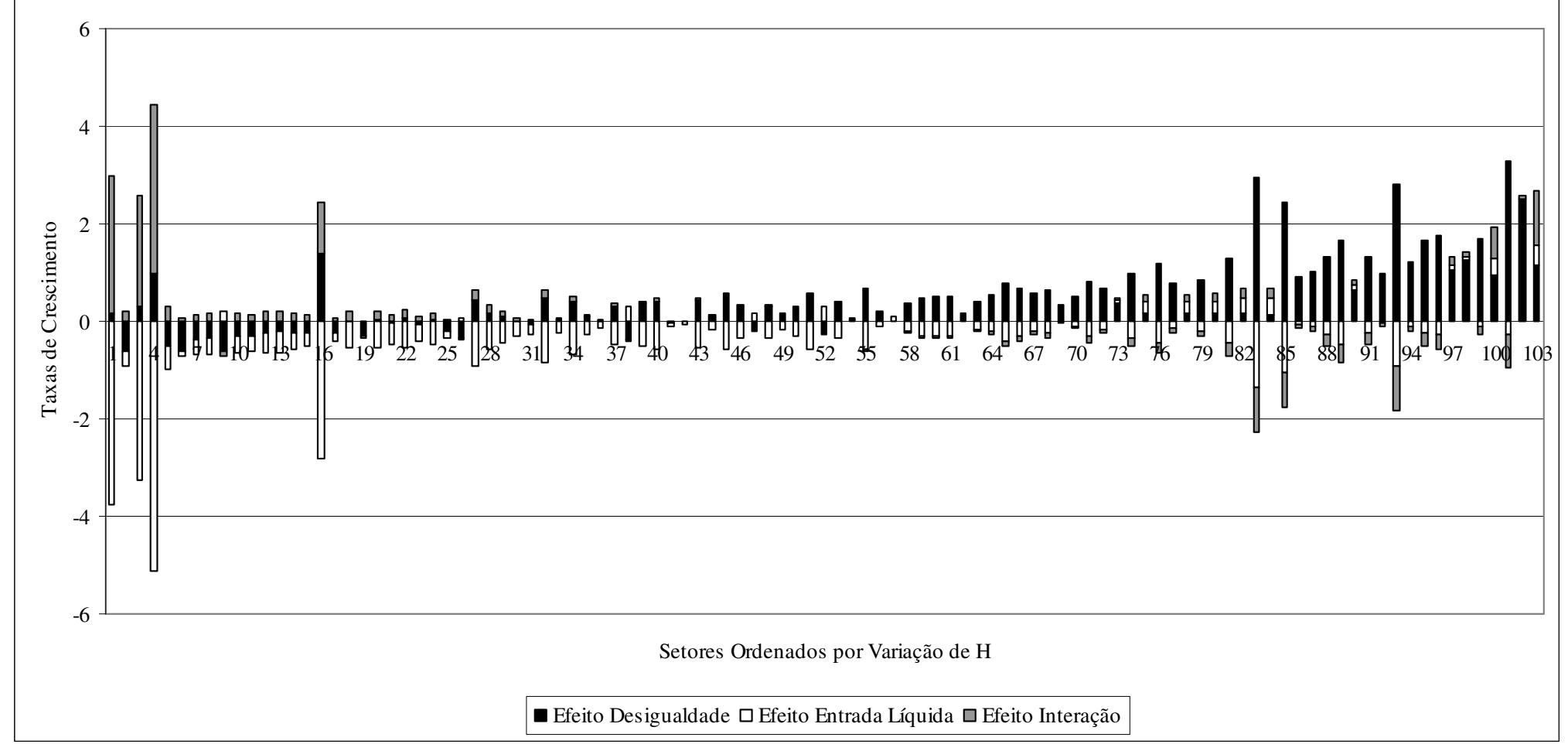

Fonte: Elaboração própria a partir de IBGE - Pesquisa Industrial Anual. 
Frederico Rocha

\section{Conclusões}

O objetivo deste artigo foi estudar a variação na concentração dos setores a três dígitos CNAE da indústria de transformação e mineração no Brasil. O foco da análise ressaltou a concentração como uma variável que consolida dinamicamente as características prevalecentes na indústria. Os resultados obtidos sugerem que a indústria de mineração e transformação sofreu, na média, reduzida mudança em seu nível de concentração. Contudo, em um determinado grupo de indústrias, as mudanças no nível de concentração foram substanciais.

Apesar da forte entrada de empresas em todos os segmentos, os resultados apresentados neste artigo assinalam que essa entrada não foi necessariamente acompanhada de um decréscimo do nível de concentração. Ao mesmo tempo, tais resultados apontam para o efeito desigualdade como tendo um papel mais destacado na determinação das mudanças na concentração de mercado. A decomposição dos efeitos em desigualdade e entrada líquida mostra que existe forte correlação entre o efeito desigualdade e a variação do índice $H$, mas a correlação entre $H$ e o efeito entrada líquida é bem mais fraca.

Ao mesmo tempo, pôde-se identificar uma correlação negativa entre o efeito entrada líquida e o efeito desigualdade. Essa evidência e as estatísticas que comprovam, no geral, um incremento superior do número de empresas com menos de 30 empregados sugerem que a maior parte da entrada ocorreu em pequena escala. Portanto, pode ter prevalecido o efeito indireto do número de empresas sobre a média da parcela de mercado das empresas em cada setor analisado, o que implica, coteris paribus, um aumento do efeito desigualdade. Ao mesmo tempo, um exame qualitativo dos segmentos de maior variação da concentração parece induzir à conclusão de que o papel das fusões e aquisições esteve longe de ser desprezível no período.

\section{Bibliografia}

BAJO, O.; SALAS, R. Inequality foundations of concentration measures. An application of the Hannah-Kay Indices. Spanish Economic Review, 4, p. 311-316, 2002.

Decomposing changes in industry concentration. The Empirical Economic Letters, v. 3, n. 6, p. 311-319, 2004.

CURRY, B.; GEORGE, D. Industrial concentration: a survey. The Journal of Industrial Economics, v. 31, n. 3, p. 203-255, 1983.

DIXIT, A. The role of investment in entry deterrence. Economic Journal, 27, p. 95-106, 1980.

DONSIMONI, M. P.; GEROSKI, P.; JACQUEMIN, A. Concentration indices and market power: two views. The Journal of Industrial Economics, v. 32, n. 4, p. 419-434, 1984.

FERREIRA, P.; ROSSI, J. New evidence from Brazil on trade liberalization and productivity growth. International Economic Review, v. 44, n. 4, p. 1383-1405, 2003. 
GORT, M. Analysis of stability and changes of market shares. The Journal of Political Economy, v. 71, n. 1, p. 51-63, 1965.

GORT, M.; KLEPPER, S. Time paths in the diffusion of product innovation. The Economic Journal, v. 92, n. 3, p. 630-653, 1982.

GROSSACK, I. Towards an integration of static and dynamics measures of industrial concentration. The Review of Economics and Statistics, v. 47, n. 3, p. 301-308, 1965.

HALL, M.; TIDEMAN, N. Measures of concentration. Journal of the American Statistical Association, v. 62, n. 317, p. 162-168, 1987.

HANNAH, L.; KAY, J. Concentration in modern industry. London: Macmillan, 1977.

HAY, D.; MORRIS, D. Industrial economics and organization: theory and evidence. Oxford: Oxford University Press, 1991.

LADERMAN, E. Changes in the structure of urban banking markets in the West. Economic Review of the Federal Bank of San Francisco, n. 1, p. 21-34, 1995.

LIEBESKIND, J. P.; OPLER, T. C.; HATFIELD, D. Corporate restructuring and the consolidation of US industry. The Journal of Industrial Economics, v. 44, p. 53-68, Mar. 1996.

MORTIMORE, M.; PERES, W. La competitividad industrial en América Latina y Caribe. Revista de la Cepal, 74, ago. 2001.

PRYOR, F. The evolution of competition in US manufacturing. Review of Industrial Organization, 9, p. 695-714, 1994. 326,2001

New trends in US concentration. Review of Industrial Organization, 18, p. 301-

. News from the monopoly front: changes in industrial concentration, 1992-1997. Review of Industrial Organization, 20, p. 183-185, 2002.

ROCHA, F. Impactos das fusões e aquisições sobre a concentração industrial: observações preliminares sobre o caso brasileiro, 1996-2000. Economia Aplicada, v. 8, n. 2, p. 255275, 2004.

; KUPFER, D. Structural changes and specialization in Brazilian industry: the evolution of the leading companies and the M\&A process. Developing Economies, v. 40, n. 4, p. 497-521, 2002.

SCHERER, F.; ROSS, D. Market structure and economic performance. Boston: Houghon Mifflin, 1990.

SCHMALENSEE, R. Inter-industry studies of structure and performance. In: SCHMALENSEE, R.; WILLIG, R. Handbook of industrial organization. North-Holland, Amsterdam, 1989.

SCHOR, A. Productivity: competition, embodied technology and heterogeneous response to tariff reduction. Evidence from Brazilian manufacturing industries. Jun. 2003. NBER Working Paper, n. W10544. Disponível em: http://papers.ssrn.com/sol3/papers.cfm?abstract_id=556531.

TIROLE, J. The theory of industrial organization. Cambridge: MIT Press, 1988. 


\section{Anexo}

\begin{tabular}{|c|c|c|c|c|c|c|c|}
\hline \multirow[b]{2}{*}{ COD } & \multirow[b]{2}{*}{ Setor } & \multicolumn{2}{|c|}{ Índice de Herfindahl } & \multicolumn{3}{|c|}{ Decomposição da Taxa de Crescimento } & \multirow[b]{2}{*}{$\begin{array}{l}\text { Variação da } \\
\text { H }\end{array}$} \\
\hline & & 1996 & 2003 & $\begin{array}{c}\text { Efeito } \\
\text { Desigualdade }\end{array}$ & $\begin{array}{c}\text { Efeito } \\
\text { Entrada } \\
\text { Líquida }\end{array}$ & $\begin{array}{c}\text { Efeito } \\
\text { Interação }\end{array}$ & \\
\hline 100 & Extração de carvão mineral & 0,1353 & 0,1136 & 0,3991 & $-0,6667$ & 0,107 & $-0,1605$ \\
\hline 112 & $\begin{array}{l}\text { Atividades de serviços relacionados com extração de } \\
\text { petróleo e gás }\end{array}$ & 0,2105 & 0,1316 & 1,3873 & $-2,8182$ & 1,0561 & $-0,3748$ \\
\hline 131 & Extração de minério de ferro & 0,4062 & 0,4009 & $-0,1944$ & 0,1837 & $-0,0024$ & $-0,0131$ \\
\hline 132 & Extração de minerais metálicos não-ferrosos & 0,13 & 0,382 & 0,9584 & 0,3333 & 0,6459 & 1,9376 \\
\hline 141 & Extração de pedra, areia e argila & 0,0083 & 0,0082 & 0,3219 & $-0,3372$ & 0,0039 & $-0,0115$ \\
\hline 142 & Extração de outros minerais não-metálicos & 0,0677 & 0,0349 & $-0,317$ & $-0,3233$ & 0,1564 & $-0,4839$ \\
\hline 151 & Abate e preparação de produtos de carne e de pescado & 0,0299 & 0,032 & 0,6795 & $-0,5695$ & $-0,0399$ & 0,0701 \\
\hline 152 & Processamento, preservação e produção de conservas & 0,0476 & 0,0646 & 0,4943 & $-0,1002$ & $-0,0359$ & 0,3583 \\
\hline 153 & Produção de óleos, gorduras vegetais e animais & 0,08 & 0,1608 & 1,2039 & $-0,0964$ & $-0,0974$ & 1,0101 \\
\hline 154 & Laticínios & 0,0672 & 0,0231 & $-0,4934$ & $-0,4739$ & 0,311 & $-0,6563$ \\
\hline 155 & Moagem, fabricação de produtos amiláceos & 0,0194 & 0,0131 & $-0,0202$ & $-0,445$ & 0,1432 & $-0,3219$ \\
\hline 156 & Fabricação e refino de açúcar & 0,0197 & 0,0475 & 1,2386 & 0,0719 & 0,1015 & 1,4119 \\
\hline 157 & Torrefação e moagem de café & 0,0678 & 0,0473 & $-0,1917$ & $-0,1575$ & 0,0475 & $-0,3017$ \\
\hline 158 & Fabricação de outros produtos alimentícios & 0,0136 & 0,0485 & 2,5094 & 0,0157 & 0,0402 & 2,5652 \\
\hline 159 & Fabricação de bebidas & 0,0388 & 0,1303 & 3,2909 & $-0,2776$ & $-0,6548$ & 2,3584 \\
\hline 160 & Fabricação de produtos do fumo & 0,3258 & 0,2241 & $-0,0762$ & $-0,3433$ & 0,1072 & $-0,3123$ \\
\hline 171 & Beneficiamento de fibras têxteis naturais & 0,0859 & 0,0802 & 0,0024 & $-0,0733$ & 0,0048 & $-0,0661$ \\
\hline 172 & Fiação & 0,0333 & 0,0258 & $-0,0798$ & $-0,188$ & 0,0424 & $-0,2254$ \\
\hline
\end{tabular}

Continua... 


\section{Continuação}

\begin{tabular}{|c|c|c|c|c|c|c|c|}
\hline \multirow[b]{2}{*}{$\mathrm{COD}$} & \multirow[b]{2}{*}{ Setor } & \multicolumn{2}{|c|}{ Índice de Herfindahl } & \multicolumn{3}{|c|}{ Decomposição da Taxa de Crescimento } & \multirow[b]{2}{*}{$\begin{array}{c}\text { Variação da } \\
\text { H }\end{array}$} \\
\hline & & 1996 & 2003 & $\begin{array}{c}\text { Efeito } \\
\text { Desigualdade }\end{array}$ & $\begin{array}{l}\text { Efeito } \\
\text { Entrada } \\
\text { Líquida }\end{array}$ & $\begin{array}{c}\text { Efeito } \\
\text { Interação }\end{array}$ & \\
\hline 173 & Tecelagem - inclusive fiação e tecelagem & 0,0199 & 0,046 & 1,0484 & 0,1145 & 0,1503 & 1,3131 \\
\hline 174 & Fabricação de artefatos têxteis, incluindo tecelagem & 0,0905 & 0,1511 & 2,9399 & $-1,3596$ & $-0,9105$ & 0,6697 \\
\hline 175 & Acabamento em fios, tecidos e artigos têxteis (p/ terc.) & 0,0202 & 0,0211 & $-0,2686$ & 0,2975 & 0,0122 & 0,0412 \\
\hline 176 & Fabricação de artefatos têxteis a partir de tecidos & 0,0111 & 0,0131 & 0,1855 & $-0,0047$ & $-0,0008$ & 0,18 \\
\hline 177 & Fabricação de tecidos e artigos de malha & 0,0244 & 0,0306 & 0,5296 & $-0,2185$ & $-0,0558$ & 0,2553 \\
\hline 181 & Confecção de artigos do vestuário & 0,0033 & 0,0034 & 0,3957 & $-0,3368$ & $-0,0148$ & 0,044 \\
\hline 182 & Fabricação de acessórios do vestuário & 0,0177 & 0,0277 & 0,8554 & $-0,1893$ & $-0,106$ & 0,56 \\
\hline 191 & Curtimento e outras preparações de couro & 0,0135 & 0,0258 & 0,9958 & $-0,0445$ & $-0,0406$ & 0,9107 \\
\hline 192 & Fabricação de artigos para viagem & 0,0214 & 0,0271 & 0,7642 & $-0,3959$ & $-0,1045$ & 0,2638 \\
\hline 193 & Fabricação de calçados & 0,0169 & 0,0127 & 0,1848 & $-0,5707$ & 0,1402 & $-0,2457$ \\
\hline 201 & Desdobramento de madeira & 0,0041 & 0,0034 & 0,0334 & $-0,2509$ & 0,0436 & $-0,1739$ \\
\hline 202 & Fabricação de produtos de madeira, cortiça & 0,0114 & 0,0175 & 1,1891 & $-0,428$ & $-0,2281$ & 0,533 \\
\hline 211 & Fabricação de celulose e outras pastas & 0,1695 & 0,3088 & 1,6709 & $-0,4667$ & $-0,3832$ & 0,8211 \\
\hline 212 & Fabricação de papel, papelão liso, cartolina & 0,0642 & 0,1188 & 0,6314 & 0,1184 & 0,1007 & 0,8505 \\
\hline 213 & Fabricação de embalagens de papel ou papelão & 0,0319 & 0,0573 & 1,0063 & $-0,1168$ & $-0,093$ & 0,7964 \\
\hline 214 & Fabricação de artefatos diversos de papel, papelão & 0,0364 & 0,0217 & $-0,2244$ & $-0,2999$ & 0,1209 & $-0,4033$ \\
\hline 221 & Edição; edição e impressão & 0,0151 & 0,0132 & 0,0042 & $-0,1495$ & 0,0189 & $-0,1264$ \\
\hline 222 & Impressão e serviços conexos para terceiros & 0,014 & 0,014 & 0,1689 & $-0,1715$ & 0,0004 & $-0,0023$ \\
\hline 223 & Reprodução de materiais gravados & 0,0985 & 0,1543 & 0,1618 & 0,2588 & 0,1469 & 0,5675 \\
\hline 232 & Fabricação de produtos derivados do petróleo & 0,9031 & 0,8435 & 0,4302 & $-0,5313$ & 0,0351 & $-0,066$ \\
\hline 234 & Produção de álcool & 0,0108 & 0,0182 & 0,1418 & 0,3198 & 0,217 & 0,6786 \\
\hline 241 & Fabricação de produtos químicos inorgânicos & 0,0279 & 0,0431 & 0,7776 & $-0,1525$ & $-0,0827$ & 0,5424 \\
\hline
\end{tabular}


Frederico Rocha

\begin{tabular}{|c|c|c|c|c|c|c|c|}
\hline \multicolumn{8}{|c|}{ Continuação } \\
\hline \multirow[b]{2}{*}{$\mathrm{COD}$} & \multirow[b]{2}{*}{ Setor } & \multicolumn{2}{|c|}{ Índice de Herfindahl } & \multicolumn{3}{|c|}{ Decomposição da Taxa de Crescimento } & \multirow[b]{2}{*}{$\begin{array}{c}\text { Variação da } \\
\text { H }\end{array}$} \\
\hline & & 1996 & 2003 & $\begin{array}{c}\text { Efeito } \\
\text { Desigualdade }\end{array}$ & $\begin{array}{l}\text { Efeito } \\
\text { Entrada } \\
\text { Líquida }\end{array}$ & $\begin{array}{c}\text { Efeito } \\
\text { Interação }\end{array}$ & \\
\hline 242 & Fabricação de produtos químicos orgânicos & 0,0709 & 0,1401 & 2,8092 & $-0,9274$ & $-0,9055$ & 0,9764 \\
\hline 243 & Fabricação de resinas e elastômeros & 0,0583 & 0,0576 & 0,3329 & $-0,3509$ & 0,0047 & $-0,0133$ \\
\hline 244 & Fabricação de fibras, fios, cabos e filamentos & 0,2604 & 0,1669 & $-0,2497$ & $-0,1707$ & 0,0613 & $-0,3592$ \\
\hline 245 & Fabricação de produtos farmacêuticos & 0,0178 & 0,0202 & 0,3683 & $-0,2058$ & $-0,0277$ & 0,1347 \\
\hline 246 & Fabricação de defensivos agrícolas & 0,091 & 0,1088 & 0,3981 & $-0,1695$ & $-0,0331$ & 0,1955 \\
\hline 247 & Fabricação de sabões, detergentes, produtos & 0,1474 & 0,1028 & 0,0272 & $-0,4733$ & 0,1433 & $-0,3027$ \\
\hline 248 & Fabricação de tintas, vernizes, esmaltes, lacas & 0,0439 & 0,0241 & $-0,1999$ & $-0,4582$ & 0,2068 & $-0,4513$ \\
\hline 249 & Fabricação de produtos e preparados químicos & 0,0299 & 0,0169 & $-0,2294$ & $-0,362$ & 0,1572 & $-0,4342$ \\
\hline 251 & Fabricação de artigos de borracha & 0,0798 & 0,0534 & $-0,3037$ & $-0,04$ & 0,0132 & $-0,3305$ \\
\hline 252 & Fabricação de produtos de material plástico & 0,0051 & 0,0046 & 0,3683 & $-0,5214$ & 0,0525 & $-0,1007$ \\
\hline 261 & Fabricação de vidro e de produtos do vidro & 0,0853 & 0,084 & 0,5627 & $-0,5863$ & 0,0087 & $-0,0149$ \\
\hline 262 & Fabricação de cimento & 0,0545 & 0,0834 & 0,1748 & 0,2326 & 0,1234 & 0,5308 \\
\hline 263 & Fabricação de artefatos de concreto, cimento- & 0,0201 & 0,0203 & 0,5797 & $-0,5668$ & $-0,0046$ & 0,0082 \\
\hline 264 & Fabricação de produtos cerâmicos & 0,0187 & 0,0247 & 0,6544 & $-0,2534$ & $-0,0811$ & 0,3199 \\
\hline 269 & Aparelhamento de pedras e fabricação de cal & 0,0224 & 0,0146 & 0,0099 & $-0,5434$ & 0,1878 & $-0,3457$ \\
\hline 271 & Produção de ferro-gusa e de ferroligas & 0,1278 & 0,0389 & 0,2974 & $-3,2632$ & 2,2701 & $-0,6957$ \\
\hline 272 & Siderurgia & 0,0882 & 0,0884 & 0,3177 & $-0,316$ & $-0,0004$ & 0,0013 \\
\hline 273 & Fabricação de tubos - exceto em siderúrgicas & 0,0511 & 0,0796 & 0,1747 & 0,246 & 0,1372 & 0,5579 \\
\hline 274 & Metalurgia de metais não-ferrosos & 0,071 & 0,0545 & $-0,0118$ & $-0,2893$ & 0,0676 & $-0,2335$ \\
\hline 275 & Fundição & 0,0134 & 0,0326 & 1,707 & $-0,1097$ & $-0,1579$ & 1,4395 \\
\hline 281 & Fabricação de estr. metálicas e obras de caldeiraria pesada & 0,0318 & 0,0171 & $-0,3104$ & $-0,2835$ & 0,1312 & $-0,4627$ \\
\hline 282 & Fabricação de tanques, caldeiras e reservatórios & 0,0712 & 0,0344 & $-0,3765$ & $-0,2921$ & 0,1511 & $-0,5175$ \\
\hline
\end{tabular}




\begin{tabular}{|c|c|c|c|c|c|c|c|}
\hline \multicolumn{8}{|c|}{ Continuação } \\
\hline \multirow[b]{2}{*}{$\mathrm{COD}$} & \multirow[b]{2}{*}{ Setor } & \multicolumn{2}{|c|}{ Índice de Herfindahl } & \multicolumn{3}{|c|}{ Decomposição da Taxa de Crescimento } & \multirow[b]{2}{*}{$\begin{array}{c}\text { Variação da } \\
\text { H }\end{array}$} \\
\hline & & 1996 & 2003 & $\begin{array}{c}\text { Efeito } \\
\text { Desigualdade }\end{array}$ & $\begin{array}{l}\text { Efeito } \\
\text { Entrada } \\
\text { Líquida }\end{array}$ & $\begin{array}{l}\text { Efeito } \\
\text { Interação }\end{array}$ & \\
\hline 283 & Forjaria, estamparia, metalurgia do pó e serviços & 0,0056 & 0,0123 & 1,7652 & $-0,2644$ & $-0,3138$ & 1,187 \\
\hline 284 & Fabricação de artigos de cutelaria, de serralherias & 0,0305 & 0,0387 & 0,6788 & $-0,3206$ & $-0,087$ & 0,2712 \\
\hline 289 & Fabricação de produtos diversos de metal & 0,0132 & 0,0117 & 0,304 & $-0,4731$ & 0,0543 & $-0,1148$ \\
\hline 291 & Fabricação de motores, bombas, compressores & 0,0316 & 0,037 & 0,5095 & $-0,2887$ & $-0,0495$ & 0,1713 \\
\hline 292 & Fabricação de máquinas e equipamentos de uso & 0,0151 & 0,0104 & 0,0582 & $-0,5428$ & 0,1705 & $-0,3141$ \\
\hline 293 & Fabricação de tratores e de máq. e equip. agrícolas & 0,0453 & 0,0984 & 1,6746 & $-0,2324$ & $-0,272$ & 1,1702 \\
\hline 294 & Fabricação de máquinas-ferramenta & 0,0353 & 0,053 & 0,9951 & $-0,3299$ & $-0,165$ & 0,5002 \\
\hline 295 & Fabricação de máquinas e equipamentos de uso específico & 0,1168 & 0,2127 & 1,323 & $-0,2759$ & $-0,2264$ & 0,8207 \\
\hline 296 & Fabricação de outras máquinas e equipamentos & 0,0157 & 0,011 & $-0,3422$ & 0,0593 & $-0,0178$ & $-0,3007$ \\
\hline 297 & Fabricação de armas, munições e equipamentos & 0,2382 & 0,2137 & $-0,3787$ & 0,3077 & $-0,0316$ & $-0,1026$ \\
\hline 298 & Fabricação de eletrodomésticos & 0,1634 & 0,13 & 0,4786 & $-0,8594$ & 0,176 & $-0,2048$ \\
\hline 301 & Fabricação de máquinas para escritório & 0,1239 & 0,4562 & 1,1581 & 0,4138 & 1,1096 & 2,6815 \\
\hline 302 & Fabricação de máquinas e equipamentos de sist. elétr. & 0,0753 & 0,1004 & 0,3501 & $-0,0128$ & $-0,0043$ & 0,3331 \\
\hline 311 & Fabricação de geradores, transformadores e mot. elétr. & 0,1022 & 0,1329 & 0,5713 & $-0,2089$ & $-0,0626$ & 0,2998 \\
\hline 312 & $\begin{array}{l}\text { Fabricação de equipamentos para distribuição energia } \\
\text { elétrica }\end{array}$ & 0,0808 & 0,1179 & 0,6933 & $-0,1601$ & $-0,0736$ & 0,4596 \\
\hline 313 & Fabricação de fios, cabos e condutores elétricos & 0,0889 & 0,0322 & $-0,6018$ & $-0,1009$ & 0,0644 & $-0,6383$ \\
\hline 314 & Fabricação de pilhas, baterias e acumuladores & 0,2512 & 0,1242 & $-0,3273$ & $-0,36$ & 0,1819 & $-0,5054$ \\
\hline 315 & Fabricação de lâmpadas e equipamentos de iluminação & 0,082 & 0,1444 & 0,9014 & $-0,079$ & $-0,0602$ & 0,7622 \\
\hline 316 & Fabricação de material elétrico para veículos & 0,193 & 0,2259 & 0,5173 & $-0,2963$ & $-0,0505$ & 0,1705 \\
\hline 319 & Fabricação de outros equipamentos e aparelhos & 0,0275 & 0,0457 & 0,1777 & 0,2913 & 0,1928 & 0,6618 \\
\hline 321 & Fabricação de material eletrônico básico & 0,1921 & 0,1035 & $-0,2379$ & $-0,4149$ & 0,1914 & $-0,4614$ \\
\hline
\end{tabular}


Frederico Rocha

\begin{tabular}{|c|c|c|c|c|c|c|c|}
\hline \multicolumn{8}{|c|}{ Continuação } \\
\hline \multirow[b]{2}{*}{ COD } & \multirow[b]{2}{*}{ Setor } & \multicolumn{2}{|c|}{ Índice de Herfindahl } & \multicolumn{3}{|c|}{ Decomposição da Taxa de Crescimento } & \multirow[b]{2}{*}{$\begin{array}{c}\text { Variação da } \\
\text { H }\end{array}$} \\
\hline & & 1996 & 2003 & $\begin{array}{c}\text { Efeito } \\
\text { Desigualdade }\end{array}$ & $\begin{array}{l}\text { Efeito } \\
\text { Entrada } \\
\text { Líquida }\end{array}$ & $\begin{array}{l}\text { Efeito } \\
\text { Interação }\end{array}$ & \\
\hline 322 & Fabricação de aparelhos e equipamentos de telefonia & 0,1261 & 0,1859 & 0,3582 & 0,0784 & 0,0372 & 0,4738 \\
\hline 323 & Fabricação de aparelhos receptores de rádio e televisão & 0,0789 & 0,0842 & 0,0486 & 0,0169 & 0,0011 & 0,0665 \\
\hline 331 & $\begin{array}{l}\text { Fabricação de aparelhos e instrumentos médico- } \\
\text { hospitalares }\end{array}$ & 0,0331 & 0,0365 & 0,2086 & $-0,0948$ & $-0,0099$ & 0,104 \\
\hline 332 & Fabricação de aparelhos e instrumentos de medida e testes & 0,0472 & 0,0661 & 0,8278 & $-0,3054$ & $-0,1222$ & 0,4002 \\
\hline 333 & Fabricação de máq., apar. e equip. de sistemas eletrônicos & 0,051 & 0,0488 & 0,1249 & $-0,1746$ & 0,0074 & $-0,0423$ \\
\hline 334 & Fabricação de aparelhos, instrumentos e materiais óticos & 0,0463 & 0,0512 & $-0,0006$ & 0,096 & 0,0101 & 0,1056 \\
\hline 335 & Fabricação de cronômetros e relógios & 0,1105 & 0,1006 & $-0,0244$ & $-0,0714$ & 0,0064 & $-0,0894$ \\
\hline 341 & Fabricação de automóveis, caminhonetas e utilitários & 0,2516 & 0,1704 & 0,0387 & $-0,5333$ & 0,172 & $-0,3226$ \\
\hline 342 & Fabricação de caminhões e ônibus & 0,328 & 0,2451 & 0,4266 & $-0,9091$ & 0,2297 & $-0,2527$ \\
\hline 343 & Fabricação de cabines, carrocerias e reboques & 0,072 & 0,0818 & 0,4723 & $-0,297$ & $-0,0401$ & 0,1351 \\
\hline 344 & Fabricação de peças e acessórios para veículos & 0,0141 & 0,0128 & 0,4182 & $-0,5607$ & 0,0512 & $-0,0913$ \\
\hline 345 & Recondicionamento ou recuperação de motores & 0,013 & 0,0066 & $-0,599$ & 0,2088 & $-0,103$ & $-0,4932$ \\
\hline 351 & Construção e reparação de embarcações & 0,2365 & 0,0707 & $-0,6123$ & $-0,2977$ & 0,2088 & $-0,7013$ \\
\hline 352 & Construção, montagem e reparação de veículos & 0,1478 & 0,2498 & 2,4409 & $-1,0357$ & $-0,7149$ & 0,6903 \\
\hline 353 & Construção, montagem e reparação de aeronaves & 0,3816 & 0,6114 & 1,298 & $-0,4342$ & $-0,2615$ & 0,6023 \\
\hline 359 & Fabricação de outros equipamentos de transporte & 0,1569 & 0,2912 & 1,3194 & $-0,25$ & $-0,2139$ & 0,8555 \\
\hline 361 & Fabricação de artigos do mobiliário & 0,0043 & 0,0037 & 0,1033 & $-0,2747$ & 0,0369 & $-0,1345$ \\
\hline 369 & Fabricação de produtos diversos & 0,0129 & 0,0097 & 0,093 & $-0,449$ & 0,1103 & $-0,2457$ \\
\hline 371 & Reciclagem de sucatas metálicas & 0,6481 & 0,1611 & 0,1807 & $-3,75$ & 2,8179 & $-0,7514$ \\
\hline 372 & Reciclagem de sucatas não-metálicas & 0,0573 & 0,0184 & 0,9664 & $-5,1176$ & 3,4726 & $-0,6786$ \\
\hline
\end{tabular}

